\title{
Effect of music on pediatric endoscopic examinations: a randomized controlled trial
}

\section{다 (용}

\author{
Authors \\ Constanza Bay ${ }^{1}$, Romina Henriquez ${ }^{2}$, Luis Villarroel ${ }^{3}$, Juan Cristóbal Gana ${ }^{4}$
}

Institutions

1 Department of Pediatrics, Escuela de Medicina, Pontificia Universidad Católica de Chile, Chile

2 Endoscopy Unit, Center of Medical Specialties, Red de Salud UC Christus, Chile

3 Department of Public Health, Escuela de Medicina, Pontificia Universidad Católica de Chile, Chile

4 Department of Gastroenterology and Nutrition, Division of Pediatrics, Escuela de Medicina, Pontificia Universidad Católica de Chile, Chile

submitted 11.8.2020

accepted after revision 9.12.2020

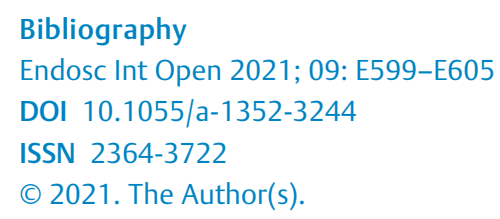

This is an open access article published by Thieme under the terms of the Creative Commons Attribution-NonDerivative-NonCommercial License, permitting copying and reproduction so long as the original work is given appropriate credit. Contents may not be used for commercial purposes, or adapted, remixed, transformed or built upon. (https://creativecommons.org/licenses/by-nc-nd/4.0/)

Georg Thieme Verlag KG, Rüdigerstraße 14,

70469 Stuttgart, Germany

Corresponding author

Dr. Juan Cristóbal Gana (JCG), Associate Professor,

Department of Gastroenterology and Nutrition, Division of

Pediatrics, Escuela de Medicina, Pontificia Universidad

Católica de Chile, Diagonal Paraguay 362, Piso 8, Santiago,

Región Metropolitana, 8330077, Chile

Fax: +56-2-2354 3402

jcgana@gmail.com

\begin{abstract}
Background and study aims The primary objective was to measure the effect of music as an adjunct to sedation in patient anxiety levels during pediatric endoscopic examinations.

Patients and methods We performed a single-blind randomized controlled trial comparing music with no music in children aged 2 to 18 years. Anxiety was measured using the Modified Yale Preoperative Anxiety Scale (m-YPAS) and the Visual Analog Anxiety Scale (VAS-anxiety). Patient perception of pain was evaluated with the Wong-Baker Faces Pain Rating Scale (WBFPRS). Patient experience, family satisfaction, and endoscopist perception of difficulty were evaluated. Sedative doses were recorded.
\end{abstract}

Results A total of 51 children were randomized to the experimental group and 49 children to the control group. The mean ages were 10.5 years and 12.3 years, respectively. There were $63 \%$ female subjects with no differences between groups. Overall, there were 85 upper endoscopies and 15 colonoscopies. In the recovery unit, the experimental group had lower average m-YPAS scores (mean score 27.7 vs $34.7 ; P<0.001)$, a higher proportion of them had low m-YPAS scores ( $80 \%$ vs $49 \% P<0.001$ ), had lower VASanxiety scores [mean score 0.55 vs $1.57(P=0.003)$ ], and had lower WBFPRS scores [mean score 2.7 vs $1.3(P=$ $0.001)]$. There were no statistically significant differences found in the amount of standard sedation given to the groups, nor in additional sedation administered. In the experimental group, the patient-reported experience was significantly better.

Conclusions The study results show that music reduces anxiety and pain associated with endoscopic procedures in children. It also facilitates these procedures and improves patient satisfaction.

\section{Introduction}

Medical examinations intended to improve the health and welfare of children may also cause them pain and anxiety. Endoscopy has become an essential diagnostic and treatment method for many gastrointestinal diseases. However, it is an invasive technique that causes anxiety in children, which sometimes leads to uncooperative patients and administration of higher levels of sedative medications. Furthermore, the number of endoscopic procedures is increased, leading to an inevitable accumulation of drug-associated adverse events (AEs), especially in young children $[1,2]$.

Although there are data to support the use of non-pharmacological techniques, their use is not standardized across prac- 
titioners and settings. Distraction has received recent attention and involves engaging children in cognitive or behavioral tasks to divert their attention from painful stimuli and reduce both pain and distress [3-6]. The contemporary definition of pain used by the International Association for the Study of Pain (IASP) is based on the multidimensional definition proposed by Melzack and Casey [7]. More recent studies have shown that these dimensions are partially dissociable, as the cognitive state of a person can modulate pain perception. Thus, distractors such as music may reduce the pain perceived $[8,9]$.

Music has been acknowledged as an inexpensive, safe, noninvasive, and effective non-pharmacological anxiolytic agent. Studies in adults have shown its effectiveness in reducing anxiety, improving patient tolerance for a procedure, and decreasing the dose of sedative medication required [10-16]. Some of these benefits have also been observed during other clinical procedures in children $[17,18]$. A meta-analysis of the effect of perioperative music in pediatric surgery demonstrated a significant positive effect on pain, anxiety, and distress [19]. In general, studies show substantial clinical heterogeneity. There is need for a more homogeneous and systematic approach to evaluate the utility of music for this specific population and identify results that are clinically applicable.

To our knowledge, this is the first study in pediatrics to evaluate the role of music as an adjunct to sedation for gastrointestinal endoscopy. The primary objective of this study was to measure the effect of music on anxiety levels during pediatric endoscopic examinations.

\section{Patients and methods}

\section{Sample and design}

The study was designed as a single-blind randomized controlled trial. It was conducted in the Endoscopy Unit of the Clinical Hospital of the Pontificia Universidad Católica of Chile. All patients between 2 and 18 years who were scheduled for outpatient endoscopic procedures were asked to participate in the study. Subjects were excluded if they had abnormal hearing, cognitive impairment, severe chronic disease, Midazolam or ketamine allergy or if the procedure was scheduled for therapy. The nurses attending the patients were not blinded to the intervention in order to conduct the study. The physician performing all the procedures was JCG, who was blinded to the music intervention. Patients were recruited from November 2014 to January 2019.

The study was approved by the Scientific Ethics Committee of the Pontificia Universidad Católica de Chile (\#14-416).

\section{Procedure}

Before the procedure, the physician made an evaluation with the scales and then left the room. The nurse then asked the patient to choose a song from a music application in an electronic device. The nurse opened the randomization envelope and if "yes" appeared, the music was turned on; if "no" appeared, the headphones were left in place without sound. She then inserted the venous line.

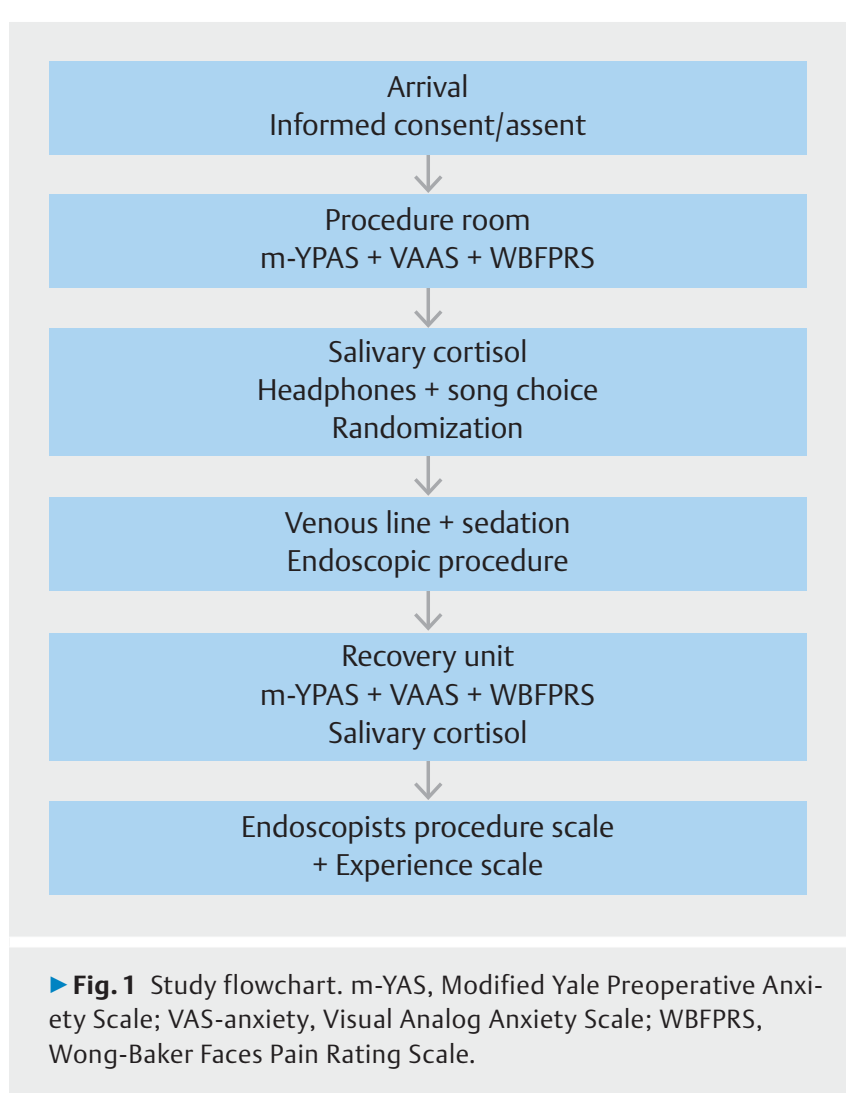

Subjects were randomly assigned to the experimental group, which included music, or to the control group, which received the routine procedure. The randomization list was generated by computer software on a 1:1 basis in blocks of 20 . Treatment allocation was concealed by using sealed opaque envelopes that were consecutively numbered. Details of the study flowchart are shown in $\mathbf{F i g . 1}$.

All subjects received nasal oxygen $(2 \mathrm{~L} / \mathrm{min})$ and standard pre-procedure intravenous sedation with Midazolam $0.1 \mathrm{mg}$ per $\mathrm{kg}$ (maximum of $1.5 \mathrm{mg}$ ) in combination with ketamine 1 $\mathrm{mg} / \mathrm{kg}$ (maximum of $15 \mathrm{mg}$ ). Patients were allowed to receive further drug administration according to procedure tolerance, with administration of a 1-mg bolus of Midazolam and $5 \mathrm{mg}$ of ketamine, always given in the same order and successively until adequate sedation had ben achieved. The nurse registered vital signs, AEs, and duration of the procedure.

Finally, in the recovery room, once awake (minimum 40 minutes after the procedure was completed), the scales were once again applied by the endoscopist together with the family and patient to assess their experience of the procedure.

\section{Measures}

The primary outcomes measured were anxiety and perception of pain experienced during the procedure. The Modified Yale Preoperative Anxiety Scale (m-YPAS) and the Visual Analog Anxiety Scale (VAS-anxiety) were used. The YPAS-m scale is an observational behavioral checklist developed by Kain et al. This instrument has good-to-excellent reliability and validity for measuring anxiety in children aged 2 to 12 years in the preo- 
perative holding area and during induction of anesthesia. A score $\geq 30$ was considered high anxiety [20]. The VAS-anxiety is a self-reported $100-\mathrm{mm}$ horizontal line scale with the two end points labeled "no anxiety or fear" and "worst possible anxiety or fear." We used it to correlate the observational findings in children aged $\geq 7$ [21].

Patient perception of pain was measured with the Wong-Baker Faces Pain Rating Scale (WBFPRS), which is validated for children aged 3 to 18 years. It is a horizontal scale of six handdrawn faces, scored from 0 to 10 , that range from a smiling "no hurt" face to a crying "hurts worst" face. A change in pain of two or more faces was considered clinically significant or having no more than minimal pain after the intervention [22].

The endoscopist assessed his perception of difficulty in performing the procedure on a scale from very easy to very difficult. Family satisfaction and patient experience were reported on a scale from very good to very bad.

\section{Statistical analysis}

\section{Sample Size}

Based on studies using the m-YPAS, we expected the control group to present an average of around 36 points on the anxiety scale at the start and end of the study, with a standard deviation equal to 12.5 (19-21). We hypothesized that the group exposed to the intervention would score on average $20 \%$ lower than the controls (ie, an average of about 28.9 points). To find significant differences between these averages, with a level of significance of $5 \%$ and a power $80 \%$, a sample of 50 patients in each group was required, meaning 100 patients total.

\section{Analysis}

The analysis of treatment effect was based on the intervention received. Differences between proportions were assessed with a Pearson chi-square test and Fisher's exact test as appropriate. Ordinal data were compared with the Wilcoxon rank-sum test. The Student's t-test was used to test our hypothesis regarding the comparison of population means between the two groups when a normal distribution was not rejected according to a Kolmogorov-Smirnov test. Analysis of covariance (ANCOVA) was used to observe the intervention effect while controlling variables that were not homogeneously distributed between the groups. All the statistical analyses were performed with STATA 14 (Stata Corporation; College Station, Texas, United States).

\section{Results}

We enrolled a total of 100 subjects, with 51 children randomized to music (experimental), and 49 children randomized to no music (control). One subject was randomized to control but received music and the analysis was performed based on the intervention received. The characteristics of the two groups of children are presented in $\mathbf{F i g . 2}$. The mean ages were 10.5 years (range 2-18 years) for the experimental group and 12.3 years (range $4-18$ years) for the control group $(P=0.045)$. This was because a greater proportion of children aged 6 or younger were in the experimental group, 13 vs 3 in the control group.

\begin{tabular}{|c|c|c|c|}
\hline & Control & Music & \\
\hline Subjects & 49 & 51 & 100 \\
\hline Age (years) & $12 \pm 3.8$ & $10.5 \pm 4.3$ & \\
\hline \multicolumn{4}{|l|}{ Sex } \\
\hline Male & 17 & 20 & 37 \\
\hline Female & 32 & 31 & 63 \\
\hline Weight (kg) & 47 & 39.6 & \\
\hline Height (mt) & 1.5 & 1.4 & \\
\hline BMI & 20 & 19 & \\
\hline Comorbidity & 14 & 12 & 26 \\
\hline Asma & 4 & 3 & \\
\hline DM1 & 1 & 2 & \\
\hline Other & 9 & 7 & \\
\hline \multicolumn{4}{|c|}{ Reason for procedure } \\
\hline Control & 4 & 6 & 10 \\
\hline Study & 45 & 45 & 90 \\
\hline \multicolumn{4}{|l|}{ Procedures } \\
\hline Endoscopy & 41 & 44 & 85 \\
\hline Colonocopy & 8 & 7 & 15 \\
\hline
\end{tabular}

Given the above, the analysis of the results was adjusted accordingly. Both groups were similar in terms of sex, anthropometry, and comorbidities. Overall, there were 85 upper endoscopies and 15 colonoscopies. There were seven colonoscopies and 44 upper endoscopies in the experimental group, and eight colonoscopies and 41 upper endoscopies in the control group. Only one patient refused to participate in the study.

\section{Modified Yale Preoperative Anxiety Scale}

The mean m-YPAS score at admission was 40.4 in the experimental group and 37.6 in the control group $(P=0.412)$. Average score at the recovery unit was 27.7 in the experimental group and 34.7 in the control group $(P<0.001)$, as shown in - Fig. 3. Overall, there were 66 high m-YPAS scores at admission, 35 in the experimental group and 31 in the control group. Of the subjects, $80 \%$ in the experimental group managed to rate a low anxiety score after the procedure (decrease from a high score and/or remain with a low score), compared to $49 \%$ in the control group $(P<0.001)$. This difference was maintained when stratifying by age and analyzing those aged $>7$ years $(P<$ $0.001)$. 


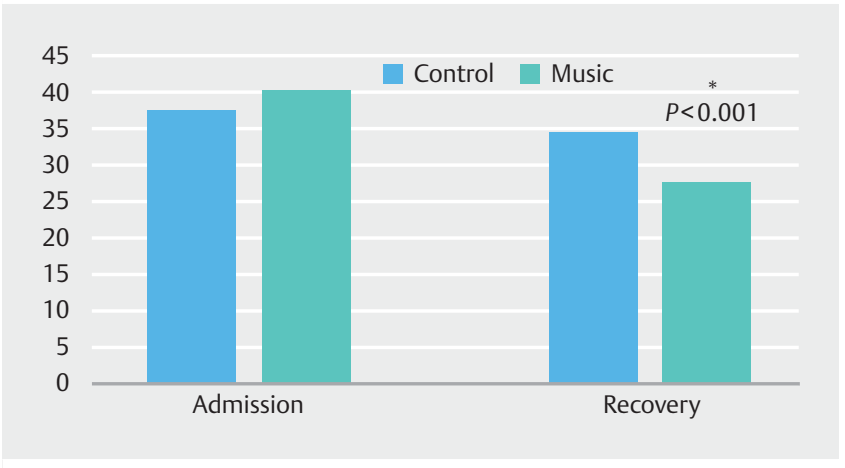

Fig. 3 m-YPAS scores at admission and recovery in both groups.

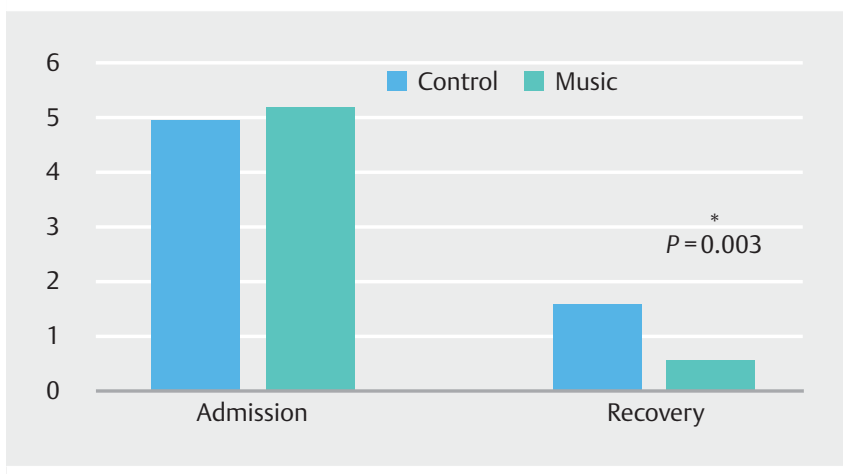

Fig. 4 VAS-anxiety scores at admission and recovery in both groups.

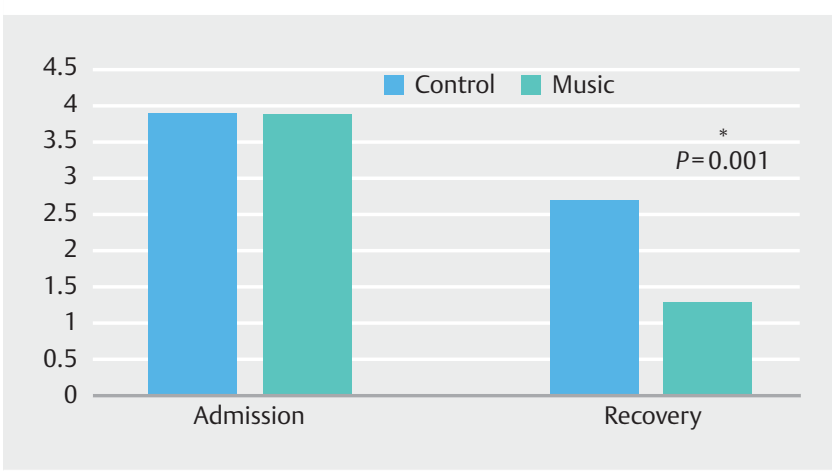

> Fig. 5 Wong-Baker Faces Pain Rating Scale scores at admission and recovery in both groups.

Regarding the type of endoscopic procedure, there were 11 patients who underwent colonoscopy with a high m-YPAS score at admission. Seven managed to rate a low score in recovery with no differences between groups $(P=0.137)$. For the patients undergoing upper digestive endoscopies, there were 55 high m-YPAS scores at admission, 26 in the control group and 29 in the experimental group. Of the experimental group, $72 \%$ managed to decrease their score to $<30$ points at the recovery unit, compared to $27 \%$ of the control group $(P<0.001)$.

\section{Visual analog anxiety scale}

This scale was applied to 84 subjects ( $>7$ years). Mean score at admission was 4.9 in the control group and 5.2 in the experimental group $(P=0.67)$. At recovery, the experimental group reported a mean score of 0.55 compared to 1.57 in the control group $(P=0.003)$, as presented in $>$ Fig. 4 . Of the 84 patients surveyed, 74 managed to reduce their score by $\geq 2$ points or report no anxiety in the recovery unit. This represented $89.5 \%$ of the experimental group and $87 \%$ of the control group $(P=$ 0.723 ). Only five patients increased their score, four of them in the control group.

\section{Wong-Baker Faces Pain Rating Scale}

This scale was applied to 99 subjects ( $>2$ years). WBFPRS mean scores at admission were 3.9 in the control group and 3.88 in the experimental group $(P=0.99)]$. At recovery, the control group reported a mean score of 2.7 whereas the experimental group reported a mean score of $1.3(P=0.001)$, as shown in - Fig. 5. Of the 99 patients surveyed, $84 \%$ of the subjects in the experimental group compared to $61 \%$ in the control group managed to decrease their previous score by two or more faces, or reported no pain at the recovery unit $(P=0.011)$.

\section{Sedation}

Subjects in the control group received Midazolam (mean 0.03 $\mathrm{mg} / \mathrm{kg} \pm 0.05 \mathrm{mg}$ ) and ketamine (mean $0.3 \mathrm{mg} / \mathrm{kg} \pm 1 \mathrm{mg}$ ), experimental group received Midazolam (mean $0.037 \mathrm{mg} / \mathrm{kg} \pm$ $0.1 \mathrm{mg}$ ) and ketamine (mean $0.4 \mathrm{mg} / \mathrm{kg} \pm 1.1 \mathrm{mg}$ ), with $P=0.28$ and $P=0.73$, respectively. Most of the patients required additional sedation with Midazolam, $82.4 \%$ of the experimental group and $85.7 \%$ of the control group $(P=0.65)$. Further additional sedation with ketamine was required in $47 \%$ of the experimental group and $38.8 \%$ of the control group $(P=0.4)$. Analyzing by the type of endoscopic procedure, of the 15 colonoscopies, eight of eight in the control group required additional sedation with Midazolam compared to five of seven in the experimental group $(P=0.104)$. Additional ketamine was required in four subjects in the control group compared to three in the experimental group ( $P=0.782)$. Regarding the upper digestive procedures, $83.5 \%$ required additional Midazolam, 34 subjects in the control group and 37 in the experimental group $(P=$ $0.885)$. Additional ketamine was administered in $42 \%$ of the procedures, 15 in the control group and 21 in the experimental group $(P=0.299)$.

\section{Duration}

The duration of the procedures was $12.2 \pm 8.5$ minutes in the control group and $11.7 \pm 6.3$ minutes in the experimental group $(P=0.73)$.

\section{Difficulty}

Physicians reported $94 \%$ of the procedures as easy or very easy to perform in the experimental group compared to $75.5 \%$ in the control group $(P=0.03)$. Also, $24.4 \%$ of the procedures in the control group were scored by the physician as being regular or difficult to perform. Whereas in the experimental group, 
only $6 \%$ were scored as regular or difficult and none of them were considered difficult, as presented in > Fig. 6.

\section{Satisfaction}

Family satisfaction with the procedure was considered good or very good in $99 \%$ of those surveyed overall, $97.9 \%$ of the control group and $100 \%$ of the experimental group $(P=0.336)$. Five patients did not answer because they were too young. Most of the patients rated the experience as being good or very good in both groups (92.6\%). The procedure was rated as very good in $45.8 \%$ of the experimental group compared to $10.6 \%$ of the control group $(P=0.001)$, as presented in $>$ Fig. 7 . Only $5 \%$ of the patients in the control group and $1 \%$ in the experimental group considered the procedure being acceptable. One patient and one family member considered the procedure as being bad regarding the patient crying, both in the control group. No one rated the endoscopic experience as being very bad.

\section{Type of music}

Subjects in the experimental group were classified by type of music. There were 13 patients who chose relaxing music and 38 patients who chose stimulating music. There was a tendency to a greater decrease in the $\mathrm{m}$-YPAS score in the relaxing group, mean change of 21.3 vs $9.7(P=0.11)$.

\section{Adverse events}

Three patients had self-limited transient hypertension, one in the control group and two in the experimental group. One patient in each group presented self-limited transient tachycardia. One patient in the experimental group desaturated up to $75 \%$, requiring oxygen support by high flow mask and momentary interruption of the procedure, but subsequently recovered and did not require other interventions. She was a healthy 4year-old who was being studied for abdominal pain. No severe AEs were reported.

\section{Discussion}

Endoscopic procedures are a stressful experience for most children. The findings from our study show that music as an adjuvant in endoscopic procedures helps to significantly decrease anxiety levels observed in patients using the m-YPAS. The experimental group had a lower final average score and a higher proportion of children with low anxiety scores $(<30)$ in the recovery unit compared with the control group. The perception of anxiety reported by patients through the VAS-anxiety scale at recovery was also significantly lower in the experimental group. The anxiety reports of children aged $>7$ years through the VAS-anxiety scale were consistent with what was reported by the endoscopist in the m-YPAS scale, showing good correlation between scales.

Inadequate pain control during medical interventions could have long-term unfavorable effects, especially among very young children. Pain and anxiety are intrinsically linked, and as children have higher levels of anxiety, they will perceive higher levels of pain [23]. Often young children confuse the feeling of anxiety with pain, so this can be a confounding factor if only

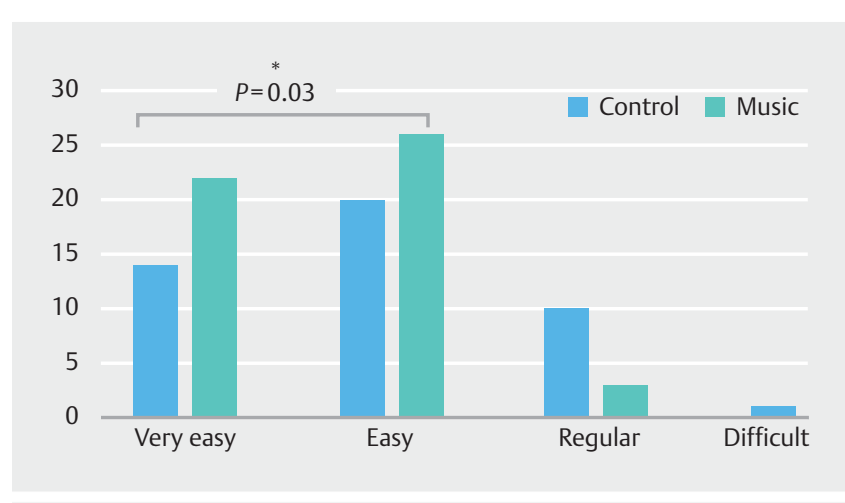

-Fig. 6 Endoscopist difficulty perception scale scores by groups.

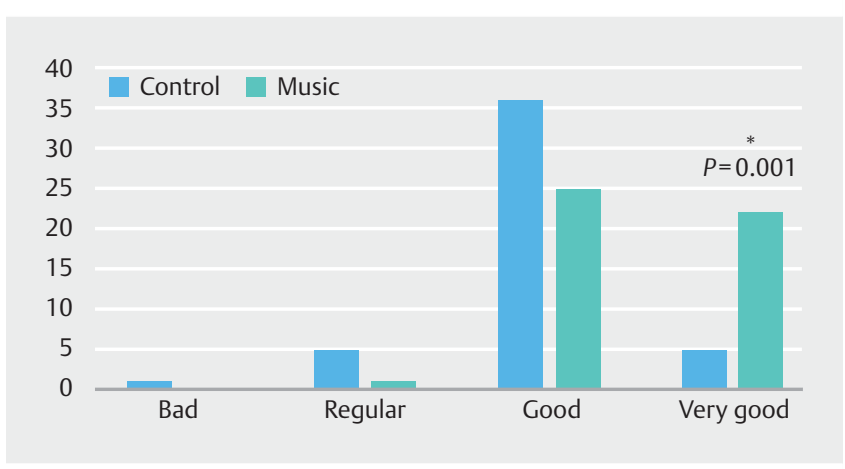

Fig. 7 Patient experience scale scores by groups.

self-reported scales are used. That is why it was decided to apply the WBFPRS, trying to achieve a more representative response in children aged $<7$ years. Results show that children in the experimental group also reported significantly lower average scores at the recovery unit.

There are plenty of studies involving music and its clinical impact on the pediatric population [17, 18]. However, studies show substantial clinical heterogeneity, as the interventions, clinical scenarios, and outcome measures vary widely across them. Studies often emphasize the need for a more homogeneous and systematic approach to evaluate the utility of music for this specific population and identify results that are clinically applicable. In this sense, the findings from our study show that music as an adjuvant in endoscopic procedures helped to significantly decrease anxiety levels. In that regard, patients not only appeared more relaxed to an observer, but they also felt less anxious. Both of these findings support the evidence that music is an effective non-pharmacologic agent that may divert attention from painful stimuli to reduce both anxiety and pain.

Among other variables studied, no statistically significant difference was found in the doses of sedatives used between the studied groups, without being able to reproduce what has been described in pediatric studies involving other procedures $[24,25]$. We found a tendency to lower requirements for sedatives in the group that chose relaxing music, but the results are likely underpowered due to the small sample size. Analysis showed no differences between groups in spite of the positive 
findings regarding anxiety and pain. That could be explained by maintaining a degree of unconsciousness during endoscopy independent of the analgesia. Moreover, endoscopies require deeper sedation than other procedures because they require a high level of immobility. Furthermore, unlike adults, children have more trouble controlling their impulses, which may favor the need for more intense stimuli to divert their attention or suppress their reactions.

Despite being blind to the intervention, the endoscopist perceived patients in the music group as less resistant and more relaxed. Less anxious patients were easier to manage in this clinical setting. This might have resulted in the perception that the procedure was easier to perform. Anxiety is probably one of the main factors that make patients more reactive to external stimuli as patients are sedated but remain conscious. Even though this benefit did not translate to a shorter duration of the particular procedures performed, it is clearly an important aspect to considerate, as it might also impact the health professional's perception of performance.

Although the overall satisfaction score was good in both groups, the results of our survey revealed that patients had a better experience when music was used. Pediatric endoscopies in our center are probably well tolerated and the patient experience is tht they are well cared for, given the high percentage of satisfaction among both patients and family. Only one patient refused to participate in the study, which underscores that this intervention is well accepted and immediately perceived as pleasant and potentially beneficial.

The cost of using music in our endoscopy unit was very low, and the benefits reported by the subjects and the physician were important. A cost-effectiveness study using music therapy-assisted procedures found that music therapy-assisted procedures in children save money, time, and staff/equipment resources in multiple procedures [24]. We did not carry out such an analysis in the present study, and one could infer that a large sample size would be required to find positive results. Nevertheless, psychological impact and wellbeing should not be underestimated. High anxiety levels may lead to an uncooperative patient and need for increased levels of sedative medications and longer procedure time. Also, unpleasant experiences in clinical settings could predispose children to feel more anxious during future procedures. This kind of intervention could be easily applicable in diverse clinical settings and may potentially lead to many benefits.

This study has several limitations. The procedures were performed in a single center and by only one endoscopist. That was the case to try to reduce intervention bias since endoscopic procedures are dependent upon who performs them. We thought that incorporating more than one endoscopist could result in variation in the doses of sedatives used and the ease and duration of the procedures, among other subjective variables. Another difficulty was the small number of colonoscopies, which did not allow for statistical analysis in this subgroup alone. Because that procedure is quite different from upper endoscopy, it would be interesting to study the two procedures separately. Regarding the results, there was a significant age difference between groups. In spite of randomization, there was a higher proportion of patients $<7$ years of age in the experimental group. This could have altered the findings, as younger children tend to be more restless and anxious. On the other hand, despite having found positive results in subjective outcomes, there were no differences between groups in the duration of the procedures nor in the administration of sedatives. The results likely are underpowered due to the small sample size, but also because endoscopies require deeper sedation than other procedures and children usually require higher doses of sedatives because their drug clearance is faster.

This was the first study to evaluate the effect of music as adjuvant therapy in pediatric patients undergoing endoscopy. It contributes to our understanding of the benefits of music during pediatric procedures and allowed us to improve the experience of the patients who participated. Based on our results, a cost-effectiveness study could be carried out to evaluate the impact of this intervention on the healthcare system. In addition, our findings could provide motivation for a much larger study involving multiple endoscopists and different centers to validate the results in these types of procedures.

\section{Conclusions}

We found that listening to music can reduce anxiety and pain in children during and after an endoscopic procedure. This evidence, therefore, should be considered when planning this type of intervention, as it may translate into an improvement in child cooperation and patient satisfaction. Children have high levels of distress and anxiety not only during endoscopy but also in anticipation of it. Therefore, prevention and reduction of distress should focus on the stages before as well as during the procedure.

\section{Acknowledgments}

The funding source for this research was the Division of Pediatrics 2014 Research Funding Competition, Pontificia Universidad Católica de Chile, Chile. The study was registered and approved by the Pontifica Universidad Católica de Chile and the Scientific Ethics Committee, number 14-416, according to local and national protocols. The authors thank the Pediatric Division of the Pontificia Universidad Católica de Chile for supporting and funding the study, all the technicians and nurses in the Endoscopy Unit at the Hospital Clínico UC Christus, and nurses Ximena García, Pía Pinochet and Bárbara Yañez.

\section{Competing interests}

The authors declare that they have no conflict of interest.

\section{References}

[1] Jevtovic-Todorovic V. Pediatric anesthesia neurotoxicity: an overview of the 2011 SmartTots panel. Anesth Analg 2011; 113: 965-968 
[2] Miller T, Park R, Sun L. Report of the Fourth PANDA Symposium on Anesthesia and Neurodevelopment in Children. J Neurosurg Anesthesiol 2014; 26: 344-348

[3] Golan G, Tighe P, Dobija N et al. Clowns for the prevention of preoperative anxiety in children: a randomized controlled trial. Pediatric Anesthesia 2009; 19: 262-266

[4] Vagnoli L, Caprilli S, Robiglio A et al. Clown doctors as a treatment for preoperative anxiety in children: a randomized, prospective study. Pediatrics 2005; 116: e563-567

[5] Murphy G. Distraction techniques for venepuncture: a review. Paediatr Nurs 2009; 21: 18-20

[6] Birnie KA, Noel M, Chambers CT et al. Psychological interventions for needle-related procedural pain and distress in children and adolescents. Cochrane Database Syst Rev 2018; 4: CD005179

[7] Melzack R, Casey KL. Sensory, motivational, and central control determinants of pain: a new conceptual model. In: Kenshalo DR. The Skin Senses. Illinois, USA: Thomas C; 1968: 423-439

[8] Moayedi M, Davis K. Theories of pain: from specificity to gate control. J Neurophysiol 2013; 109: 5-12

[9] Megel M, Houser C, Gleaves L. Children's responses to immunizations: lullabies as a distraction. Issues Compr Pediatr Nurs 1998; 21: 129145

[10] Lee DW, Chan KW, Poon CM et al. Relaxation music decreases the dose of patient-controlled sedation during colonoscopy: a prospective randomized controlled trial. Gastrointest Endosc 2002; 55: 33-36

[11] Palakanis K, DeNobile J, Sweeney W et al. Effect of music therapy on state anxiety in patients undergoing flexible sigmoidoscopy. Dis CoIon Rectum 1994; 37: 478-481

[12] Smolen D, Topp R, Singer L. The effect of self-selected music during colonoscopy on anxiety, heart rate, and blood pressure. Applied Nursing Research 2002; 15: 126-136

[13] Schiemann U, Gross M, Reuter R et al. Improved procedure of colonoscopy under accompanying music therapy. Eur J Med Res 2002; 7: 131-134

[14] Rudin D, Kiss A, Wetz RV et al. Music in the endoscopy suite: a metaanalysis of randomized controlled studies. Endoscopy 2007; 39: 507510
[15] El-Hassan H, McKeown K, Muller AF. Clinical trial: music reduces anxiety levels in patients attending for endoscopy. Aliment Pharmacol Therap 2009; 30: 718-724

[16] Costa A, Montalbano LM, Orlando A et al. Music for colonoscopy: A single-blind randomized controlled trial. Digest Liver Dis 2010; 42: 871-876

[17] Mrázová M, Celec P. A systematic review of randomized controlled trials using music therapy for children. J Altern Complement Med 2010; 16: doi:10.1089/acm.2009.0430

[18] Klassen JA, Liang $Y$, Tjosvold $L$ et al. Music for pain and anxiety in children undergoing medical procedures: a systematic review of randomized controlled trials. Ambulat Ped 2008; 8: 117-128. doi:10.1016/j.ambp.2007.12.005

[19] van der Heijden MJE, Oliai Araghi S, van Dijk M et al. The effects of perioperative music interventions in pediatric surgery: a systematic review and meta-analysis of randomized controlled trials. PLOS ONE 2015; 10: e0133608

[20] Kain ZN, Mayes LC, Cicchetti DV et al. The Yale Preoperative Anxiety Scale: how does it compare with a "gold standard"? Anesth Analg 1997; 85: 783-788

[21] Crandall M, Lammers C, Senders C et al. Initial validation of a numeric zero to ten scale to measure children's state anxiety. Anesth Analg 2007; 105: 1250-1253

[22] Tomlinson D, von Baeyer CL, Stinson JN et al. A systematic review of faces scales for the self-report of pain intensity in children. Pediatrics 2010; 126: e1168-1198

[23] Young KD. Pediatric procedural pain. Ann Emerg Med 2005; 45: 160 171

[24] DeLoach WalworthD. Procedural-support music therapy in the healthcare setting: a cost-effectiveness analysis. J Pediatr Nurs 2005; 20: $276-284$

[25] Gozal D, Gozal Y. Classical music during magnetic resonance imaging in children under propofol sedation. Cahiers D'Anesthesiol 1998; 46: 387-389 